\title{
Linear-to-Circular Polarization Converter with Adjustable Bandwidth Realized by the Graphene Transmissive Metasurface
}

\section{Xinlei Zhang}

Nanjing University of Posts and Telecommunications

Haining Ye

Nanjing University of Posts and Telecommunications

Yan Zhao

Nanjing University of Posts and Telecommunications

Haifeng Zhang ( $\square$ hanlor@163.com )

Nanjing University of Posts and Telecommunications https://orcid.org/0000-0002-9890-8345

\section{Research Article}

Keywords: Tunable polarization converter, Adjustable bandwidth, Graphene

Posted Date: November 11th, 2021

DOl: https://doi.org/10.21203/rs.3.rs-898044/v1

License: (a) (i) This work is licensed under a Creative Commons Attribution 4.0 International License.

Read Full License

Version of Record: A version of this preprint was published at Plasmonics on January 28th, 2022. See the published version at https://doi.org/10.1007/s11468-022-01598-8. 


\title{
Linear-to-circular Polarization Converter with Adjustable
}

\section{Bandwidth realized by the Graphene Transmissive Metasurface}

\author{
Xinlei Zhang ${ }^{1}$, Haining Ye $^{1}$, Yan Zhao ${ }^{1}$ and Haifeng Zhang $^{1 *}$ \\ ${ }^{1}$ College of Electronic and Optical Engineering \& College of Microelectronics, Nanjing \\ University of Posts and Telecommunications, Nanjing 210023, China
}

\begin{abstract}
A tunable linear-to-circular polarization converter (LTCPC) for the terahertz (THz) regime which consists of two conductive layers and a graphene transmissive metasurface layer separated by two dielectric layers is reported in this work. The equivalent surface resistance modeling method is adopted to investigate the peculiar electronic properties of graphene. The simulation results show that when the Fermi energy $\left(E_{\mathrm{f}}\right)$ is $1.1 \mathrm{eV}$, the linearly-polarized wave can be transformed into the circularly-polarized wave in the working band ranging from $0.9498 \mathrm{THz}$ to $1.3827 \mathrm{THz}$ (the relative bandwidth is $37.1 \%$ ) with axial ratio (AR) less than $3 \mathrm{~dB}$. Moreover, the bandwidth can be regulated to the desired one by varying the Fermi level of graphene metasurface via a bias voltage rather than manually modifying the structure. We have analyzed the mechanism of the polarization conversion, especially, the magnitudes and the phase difference of cross- and co-polarization transmission coefficients, AR curves, and surface current diagrams at $y$-polarized incidence. Our findings open up promising possibilities towards the realization of graphene controllable devices for polarization modulation, which has advantages of adjustability over traditional devices.
\end{abstract}

Keyword: Tunable polarization converter; Adjustable bandwidth; Graphene

\section{Introduction}

Manipulating the polarization states is of central interest in various fields, and the ability of which enables us to control electromagnetic (EM) waves for a wide range of applications due to the fact that many phenomena are inherently polarization-sensitive like sensors [1] and imaging [2]. According to the combination of different amplitude and phase, the polarized waves can be classified into the linear wave, circular wave, and elliptical wave among which circular polarization wave, as output wave of linear-to-circular polarization converter (LTCPC) [3], particularly offers an approach in the antennas [4], satellites [5], and liquid crystal displays [6]. Different conventional methods are employed to manipulate the polarization of EM waves including birefringent materials and chiral materials [7-8], the disadvantages of which involve bulky setup and low effectiveness. Hence, how to manipulate the EM waves flexibly and efficiently becomes the focus of current research. For instance, the plasmonic metasurfaces have been utilized to manipulate the polarization form and realized ultrathin quarter-wave plates [9].

\footnotetext{
*Corresponding author at: College of Electronic and Optical Engineering \& College of Microelectronics, Nanjing University of Posts and Telecommunications, Nanjing 210023 ,China.

E-mail: hanlor@163.com (Hai-Feng Zhang)
} 
With a series of novel EM properties [10-12], such as the negative refractive index and the circular dichroism, metamaterials break the inherent weaknesses of the conventional materials to achieve EM modulation [13] through designing unit structure reasonably and further obtain exceptional physical properties. Wide bandwidth enables the flexible manipulation of the EM waves in a wide range avoiding generating frequency offset, that is to say, polarization converters can have larger system capacity and stronger anti-interference ability, which save space cost and help to solve electromagnetic compatibility problems. A high-efficiency transmissive metasurface was put forward by $\mathrm{Li}$ et al. [14] obtained the function of LTCPC in $9.8-18.3 \mathrm{GHz}$ with a relative bandwidth (RB) of $60.5 \%$ with the formula of $2 \times\left(f_{\mathrm{H}}-f_{\mathrm{L}}\right) /\left(f_{\mathrm{H}}+f_{\mathrm{L}}\right)$. From another angle, Wang et al. [15] proposed a frequency selective surface realizing LTCPC at several resonant frequencies, which can be interpreted as a narrow bandwidth compared with the previous one.

However, devices based on the artificial metamaterial cannot fully meet the requirements of flexible performance and it is difficult to change its properties once the structure is determined [16], which gives impetus to discover the new controllable metamaterials to make the polarization converter (PC) intelligent and functional. In an attempt to enable dynamic control, like liquid crystals [17], varactor diodes are introduced as tunable load, with clear advances over currently available technology in terms of flexible control of EM waves. The essence of the tunable metamaterials is to change the spatial distribution of the equivalent dielectric constant or the equivalent magnetic conductivity of their unit structure, so as to change the transmission characteristics of the electromagnetic waves, and then realize the dynamic control of the resonance frequency. For example, the active meta-device consists of complementary electric split-ring resonators embedded with vanadium dioxide is presented [18] enabling the metasurface to achieve linear-to-circular polarization conversion at two adjustable frequency points. Despite these considerable materials, a wide range of applications are still on the way due to the limitations of existing technologies. Graphene, a two-dimensional layer with carbon atoms in a honeycomb lattice arrangement, exhibits its extraordinary potential in the aspects of optical transparency, flexibility, and electron mobility [19]. The EM parameters can be flexibly adjusted by applying a magnetic field or bias voltage to change the Fermi energy $\left(E_{\mathrm{f}}\right)$ of graphene, thereby this kind of metasurface can modify the characteristics of EM waves and realize dynamic control of the resonance frequency. Based on these characteristics, much reconfigurable polarization manipulating devices have been proposed recently [20-21]. The remarkable properties enhance the interaction between light and matter, so the graphene-based devices offer a new approach in polarization modifications, which illuminate promises for high-speed electrically controllable optoelectronic devices.

Given the fact that the current devices cannot balance between bandwidth and efficiency meantime their fabrication is still complicated, Cheng et al. proposed a tunable optical PC composed of asymmetric graphene nanocrosses, but the bandwidth was limited [22]. A structure consists of periodic $\phi$-shaped graphene sheets was put forward, but it lacks discussion about tunability [23]. Although the tuning process is a more intricate issue, the advantage of using graphene as tuning elements is very attractive, so in order to get with the demands of easier-to-implement polarizers, the present work should look into the design of bandwidth adjustable PC. For instance, Zhang successfully proposed a polarization converter but the tuning effect is not obvious on which we are going to make improvement [24].

A new approach to realize a tunable LTCPC is presented in this paper by inserting the oblique 
graphene stripes in the middle of the metal-dielectric-metal sandwich structure. The strong tunable characteristic of graphene is earned by adjusting the $E_{\mathrm{f}}$, together with the coupling of the metallic structures, enables the phase and polarization modified for the transmitted waves and brings about a broad tuning of the bandwidth. Under the situation of $E_{\mathrm{f}}=1.1 \mathrm{eV}$, this setup can transform linearly $y$-polarized waves into circularly polarized waves in the transmission mode, meanwhile, the axis ratio (AR) of the LTCPC is lower than $3 \mathrm{~dB}$ over a wide frequency ranging from 0.9498 $\mathrm{THz}$ to $1.3827 \mathrm{THz}$. By electrically doping the graphene chemical potential naturally from $0.2 \mathrm{eV}$ up to $1.1 \mathrm{eV}$, the frequency band can be dynamically expanded without modifying the physical geometry. This result brings to light the remarkable properties of graphene in the term of bandwidth adjustment and polarization conversion efficiency. It should be emphasized that this article predominantly focuses on theoretical research, and how to verify those results by the experiments is beyond the scope of this article.

\section{Design and simulation}

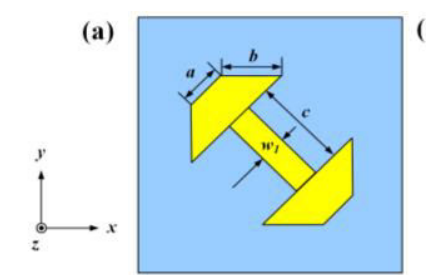

(b)
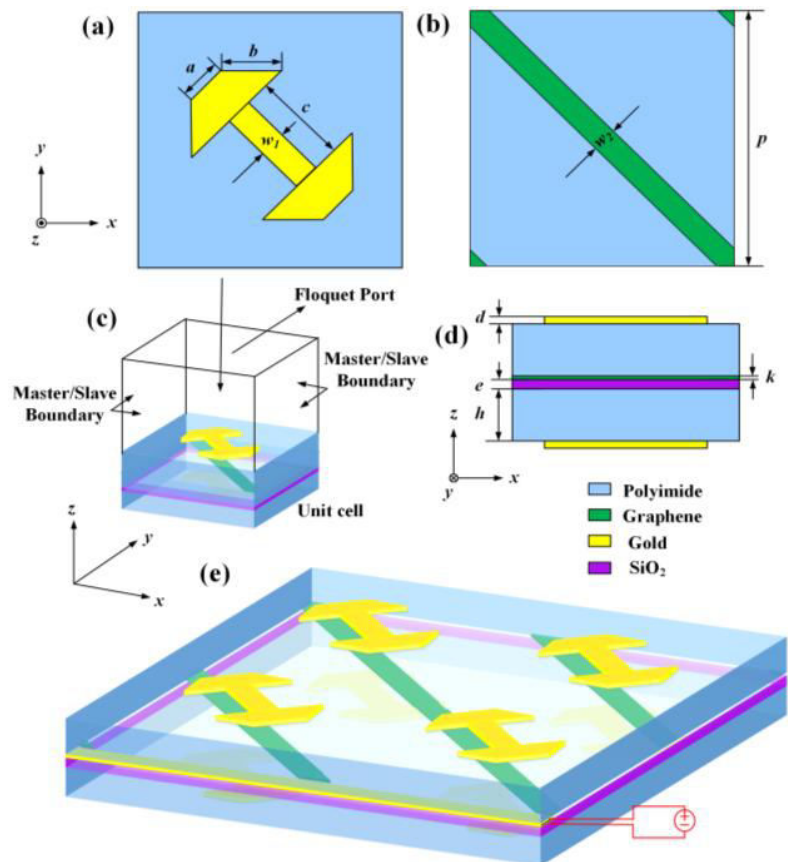

Fig.1. Schematic diagrams of the proposed LTCPC unit cell: (a) the front view of the top or bottom conductive layer, (b) the front view of the middle graphene layer, (c) the schematic diagram of the port setting and boundary conditions for the simulation, (d) the side view and (e) the periodic array stereogram.

\begin{tabular}{|c|c|c|c|c|c|}
\hline \multicolumn{7}{|c|}{ Table 1. The geometries of the designed PC. } \\
\hline Parameters & $a$ & $b$ & $c$ & $p$ & $w_{1}$ \\
\hline Value $(\mu \mathrm{m})$ & 23 & 26 & 42 & 120 & 10 \\
\hline Parameters & $w_{2}$ & $d$ & $k$ & $h$ & $\mathrm{e}$ \\
\hline Value $(\mu \mathrm{m})$ & 10 & 0.2 & 0.00034 & 17 & 0.1 \\
\hline
\end{tabular}

The unit cell of the proposed LTCPC contains three metasurfaces separated by two dielectric layers as shown in Fig.1. The geometric shapes and parameters of two gold resonances at the top and bottom layers are completely the same as displayed in Fig.1(a). While the middle layer is totally different, which is provided with oblique graphene stripes (width $w_{2}$ is $10 \mu \mathrm{m}$ ), one is set 
beneath the gold resonant unit and another is set under the gap area and does not spatially overlap gold resonant units as shown in Fig.1(b). The side view of the proposed 5-layers sandwich structure is plotted in Fig.1(d). The top and bottom resonant layers both contain an arrow-shaped gold resonant unit with a pair of corners truncated whose conductivity is $4.561 \times 10^{7} \mathrm{~S} / \mathrm{m}$. A $h=17$ $\mu \mathrm{m}$ thick polyimide dielectric spacer with the relative permittivity $\varepsilon_{r}$ of 3.5 and the loss tangent $\tan \delta$ of 0.0027 is considered as a separation layer. After optimal selection, the detailed parameters of the unit cell are listed in Table 1.

Under the applied magnetic field or bias voltage, the electrical conductivity of graphene can be flexibly adjusted and the tunable effect generated in graphene material does well to attain tunability properties in the proposed LTCPC which breaks the detuning defect of traditional devices. Hence, in order to uniformly control the conductivity of graphene stripes, we linked the graphene stripes together with a metal electrode rather than as separate parts. Below the graphene sheet, a thin silicon dioxide $\left(\mathrm{Sio}_{2}\right)$ layer with permittivity $\varepsilon_{r}=3.9$ and the thickness $e=0.1 \mu \mathrm{m}$ is used to load static bias voltage [25]. The special connection mode is depicted in Fig.1(e) that a gold electrode is added at the edges of the periodic array in direct contact with the oblique graphene stripes, which is responsible for gate voltage in its real applications [26]. Under these circumstances, the $E_{\mathrm{f}}$ of all graphene stripes in the middle layer can be effectively and simultaneously tuned by the applied gate voltage whose conductive contacts are respectively linked to the metal electrode and $\mathrm{Sio}_{2}$ layer with an adopted excitation probe. In the simulations to analyze the characteristic of the proposed LTCPC, as depicted in Fig.1(c), the open condition along the $z$-axis for inputting and detecting THz plane waves and the $x$-axis and $y$-axis directions are set as Master/Slave boundary conditions. The self-adaption method makes the mesh size of the graphene layer small enough for an accurate simulation result. Besides, many units form such a large periodic array and the influences of the added gold electrode and excitation probe are so tiny that can be ignored [27-28]. Thus, this model can be used as a periodic array to be carried out in software HFSS with the boundary conditions that met the requirements. Taking into consideration that the thickness of graphene is only $0.34 \mathrm{~nm}$, so the extremely thin influence of the edge effect can be overlooked, and the graphene metasurface can be equivalent to an infinitely thin two-dimensional planar material. All in all, the surface conductivity $(\sigma)$ can be calculated to represent the electro-optical properties of graphene. As an isotropic medium, $\sigma$ can be inferred from the Kubo formula [29] without taking into account the bias of the electric and magnetic fields:

$$
\sigma(\omega)=\sigma_{\text {intra }}(\omega)+\sigma_{\text {inter }}(\omega)
$$

The formula includes intraband and interband parts, which can be expanded into: [30]

$$
\sigma(\omega)=\frac{i e^{2}}{\pi \hbar^{2}} \frac{E_{\mathrm{f}}}{\omega+i \tau^{-1}}-\frac{i e^{2}}{4 \pi \hbar^{2}} \ln \left[\frac{2 E_{\mathrm{f}}+\hbar\left(\omega+i \tau^{-1}\right)}{2 E_{\mathrm{f}}-\hbar\left(\omega+i \tau^{-1}\right)}\right]
$$

Assuming that the proposed LTCPC is operated in the THz region, where intraband parts play the important role compared with interband parts, hence $\sigma$ can be simplified to:

$$
\sigma(\omega)=\frac{i e^{2}}{\pi \hbar^{2}} \frac{E_{\mathrm{f}}}{\omega+i \tau^{-1}}
$$

where $E_{\mathrm{f}}$ is chemical potential tuned by an external gate voltage, $e$ is the electron charge, $\hbar=h /(2 \pi)$ is the simplified Plank constant, and $\omega$ represents the angular frequency. Besides, $\tau$ expressed as 
$\mu E_{\mathrm{f}} / e v_{\mathrm{F}}^{2}$ is taken as the carrier scattering rate, where $\mu$ and $v_{\mathrm{F}}$ defined as the carrier mobility and the Fermi velocity $\left(v_{\mathrm{F}} \approx c / 300\right)$, respectively.

In order to explore the practical uses of graphene more deeply, accurate numerical calculation methods are applied to explore the physical understanding of the tuning mechanism. When modeling such device containing the graphene metasurface, the graphene usually can be equivalent to a suitable impedance surface owning to the extremely little influence of thickness. The corresponding surface resistance and surface inductance values can be obtained from the formula of $Z_{\mathrm{s}}=R_{\mathrm{s}}+j w L_{\mathrm{s}}=1 / \sigma_{\mathrm{s}}$. Taking advantage of the equivalent surface resistance modeling method to investigate the peculiar electronic properties of graphene can solve the problems aroused by the over fineness of grid division due to the thickness of graphene. The relationship between the surface impedance of graphene and $E_{\mathrm{f}}$ is shown in Fig.2.
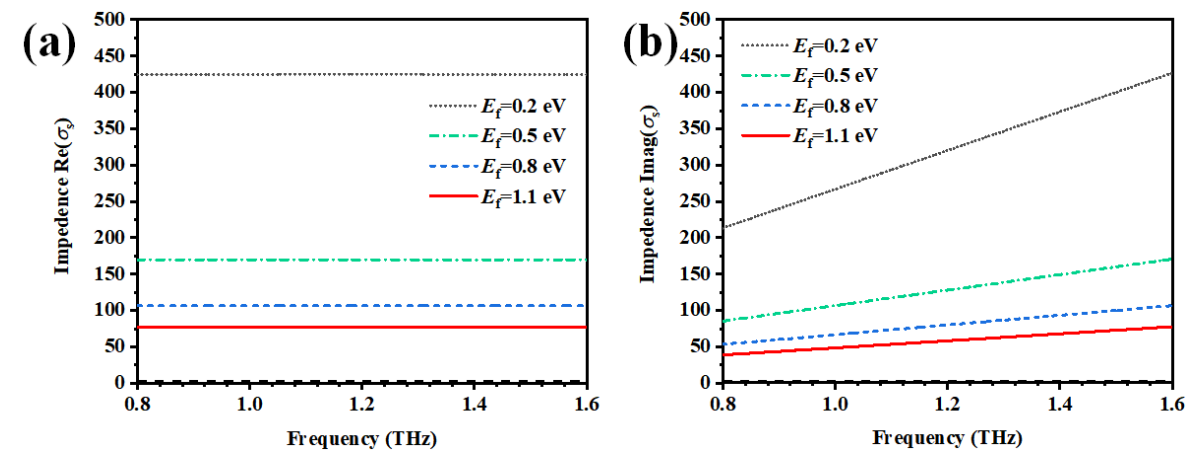

Fig.2. the real and imaginary parts when $E_{\mathrm{f}}$ ranges from $0.2 \mathrm{eV}$ to $1.1 \mathrm{eV}$, (a) The real part $\operatorname{Re}\left(\sigma_{\mathrm{s}}\right)$ of the graphene, and (b) The imaginary part $\operatorname{Imag}\left(\sigma_{\mathrm{s}}\right)$ of the graphene.

It can be inferred from the impedance model in Fig.2 that real part $\left(\operatorname{Re}\left(\sigma_{\mathrm{s}}\right)\right)$ keeps stable under a certain $E_{\mathrm{f}}$ condition over 0.8-1.6 THz band, while the imaginary part $\left(\operatorname{Imag}\left(\sigma_{\mathrm{s}}\right)\right)$ maintains a good linear relationship with frequency. The relationship indicates that the surface resistance of graphene possesses high stability in relation to the frequency and the values of the two parts drop drastically on different levels with the increasing of the chemical potential, exhibiting a remarkable tunable optical property of graphene. And it can be visually deduced that the higher the Fermi level is, the higher the conductivity is. As a result, in the simulation, by analyzing the surface impedance of graphene under different chemical potentials, high impedance surfaces based on graphene are designed and the simulation results are discussed in the following.

\section{Results and Discussions}

To evaluate the polarization conversion performance of our LTCPC more intuitively and numerically, we suppose that the $y$-polarized incident EM wave is along the $z$-direction perpendicularly. When the linearly incident EM wave impinges on the metasurface of the LTCPC, the incident wave is expressed as $E_{i}=E_{y}=\hat{y} E_{0} e^{i k_{z} z}$, and the transmitted electric field can be split into $x$ and $y$ components with the formula of $E_{\mathrm{t}}=\hat{y} T_{y y} E_{i}+\hat{x} T_{x y} E_{i}$. The co-polarization transmitted and cross-polarization transmitted coefficients are $T_{\mathrm{yy}}=t_{\mathrm{yy}} e^{i \varphi_{\mathrm{yy}}}$ and $T_{\mathrm{xy}}=t_{\mathrm{xy}} e^{i \varphi_{\mathrm{xy}}}$, where $t_{\mathrm{yy}}\left(t_{\mathrm{xy}}\right)$ and $\varphi_{\mathrm{yy}}\left(\varphi_{\mathrm{xy}}\right)$ are the amplitude and phase of transmission coefficients, respectively.

In detail, the orthotropy of the metasurface structure brings about two independent transmission coefficients $T_{\mathrm{yy}}$ and $T_{\mathrm{xy}}$, in the light of the difference and connections between the two independent transmission coefficients, the polarization state of the transmitted wave can be determined completely. A high-efficiency LTCPC must satisfy the amplitude terms that $t_{\mathrm{xy}}$ and $t_{\mathrm{yy}}$ 
are approximately equal and as high as possible. Besides, $\varphi=\varphi_{\mathrm{xy}}-\varphi_{\mathrm{yy}}$ is defined as the phase difference between the $x$ and $y$ components, and only meeting the qualification of $\varphi= \pm \pi / 2+2 \mathrm{k} \pi$ confirms that the transmitted beam is highly circularly polarized. For such a LTCPC, the cross-coupling between electric and magnetic fields exists and works at the resonance frequencies, therefore, the right-hand circularly polarized waves and left-hand circularly polarized waves encounter different transmission coefficients which mainly depend on $\varphi=+\pi / 2+2 \mathrm{k} \pi$ or $\varphi=-\pi / 2+2 \mathrm{k} \pi$, endowing the LTCPC a broad application prospect in the manufacture of the anisotropic components. Besides, the proposed structure includes the graphene metasurface, whose electromagnetic parameters can be appropriately changed by adjusting the $E_{\mathrm{f}}$. Even if the incident wave is predetermined and fixed, the strong light-graphene interaction, coupling with metal resonant units, enables a broad tuning of transmission coefficients and phase for the transmitted waves.
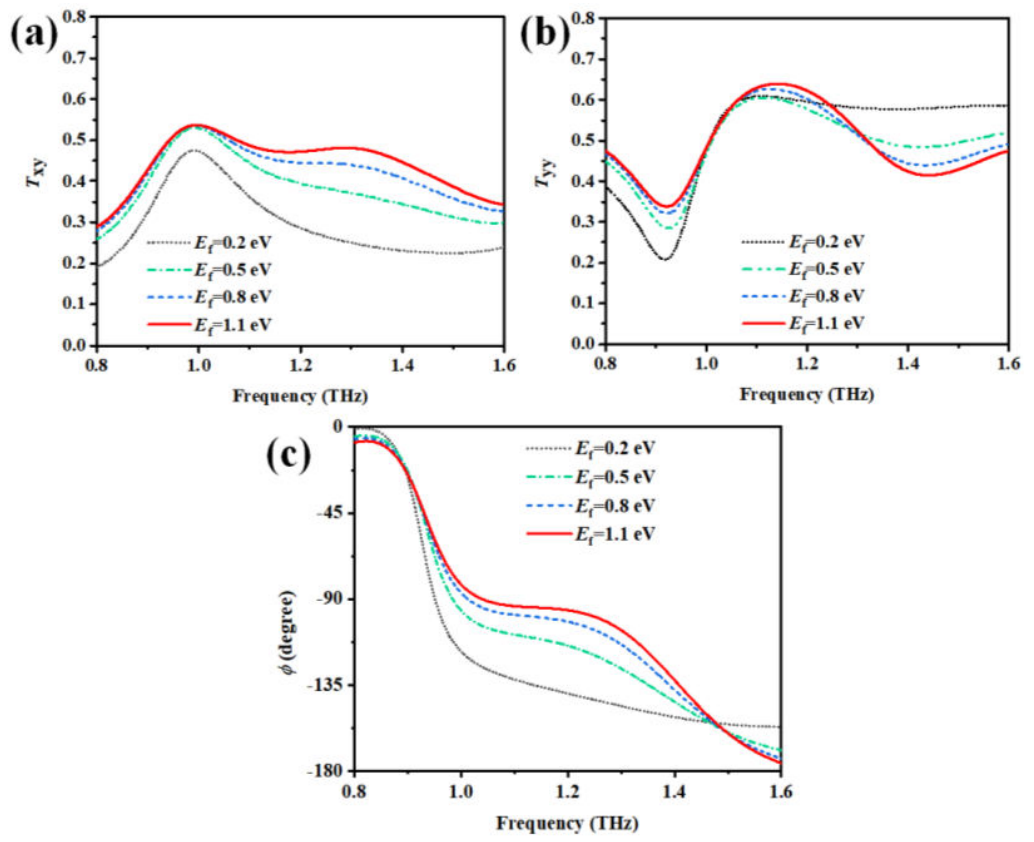

Fig.3. The simulated amplitude and phase curves of the proposed LTCPC at four $E_{\mathrm{f}}$ from $0.2 \mathrm{eV}$ to $1.1 \mathrm{eV}$ with a step of 0.3: (a) the amplitude of co-polarization, (b) the amplitude of cross-polarization, and (c) the phase difference.

Because of the orthotropy of the metasurface structure and the extraordinary ability of graphene in terms of tunability, the amplitude and phase difference of co- and cross-polarization transmission coefficients are changed step by step which is depicted in Fig.3, where different $E_{\mathrm{f}}$ curves are marked with different colors. The larger $E_{\mathrm{f}}$ is, the stronger the metallic property of graphene is, the coupling between the resonant layer is also enhanced, which leads to the big variation of the coefficients. It can be seen from Figs.3.(a) and (b) that both values of $t_{\mathrm{yy}}$ and $t_{\mathrm{xy}}$ are elevated as $E_{\mathrm{f}}$ increases from $0.2 \mathrm{eV}$ to $1.1 \mathrm{eV}$ especially in the high-frequency band and gradually reach around 0.6 throughout the whole bandwidth from 0.9498 to $1.3827 \mathrm{THz}$ when $E_{\mathrm{f}}=$ $1.1 \mathrm{eV}$. In the meantime, $\varphi$ also enhances with the increase of $E_{\mathrm{f}}$ and finally keeps close to $-\pi / 2$ throughout the working band from only the low frequency of $\varphi$ is fixed at $-\pi / 2$, which conforms to the requirements of the right-hand LTCPC. It is worth mentioning that the cross-coupling feature 
can be dynamically enhanced by the Fermi level of graphene increasing from $0.2 \mathrm{eV}$ to $1.1 \mathrm{eV}$, where graphene is gradually coupled with metal to form the new resonance leading to the broadband right-hand LTCPC. Therefore, in the following discussion, the Fermi energy $E_{\mathrm{f}}$ is fixed at $1.1 \mathrm{eV}$ for a wider bandwidth feature.

In order to evaluate the performance of the LTCPC, we introduce the four Stokes [31] parameters to describe the transmitted wave:

$$
\begin{gathered}
I=\left|t_{\mathrm{yy}}\right|^{2}+\left|t_{\mathrm{xy}}\right|^{2} \\
Q=\left|t_{\mathrm{yy}}\right|^{2}-\left|t_{\mathrm{xy}}\right|^{2} \\
U=2\left|t_{\mathrm{yy}}\right| \cdot\left|t_{\mathrm{xy}}\right| \cos \varphi \\
V=2\left|t_{\mathrm{yy}}\right| \cdot\left|t_{\mathrm{xy}}\right| \sin \varphi
\end{gathered}
$$

The ellipse angle $\beta$ and AR for the transmitted wave can be obtained from $\sin 2 \beta=\mathrm{V} / \mathrm{I}$,

$$
\begin{gathered}
\beta=\frac{1}{2} \arcsin \left(\frac{V}{I}\right) \\
A R=10 \log _{10}[\tan (\beta)] \\
\alpha=\frac{1}{2} \arctan \left(\frac{U}{Q}\right)
\end{gathered}
$$

The ellipticity angle $\beta$ stands for the shape of the ellipse and AR is taken as the figure of merit for the polarization state of transmitted beam calculated and plotted in Fig. 4 with $E_{\mathrm{f}}=1.1 \mathrm{eV}$. The polarization angle $\alpha$ represents the direction of the principal axis of the reflected polarized ellipse. In consideration, that $\beta$ and $\mathrm{AR}$ are of greater significance, so in our paper, we gave priority to these two factors, and the discussion about $\alpha$ is omitted. As shown in Fig.4(a), the simulated results qualitatively show that $\mathrm{AR}$ is less than $3 \mathrm{~dB}$ from $0.9498 \mathrm{THz}$ to $1.3827 \mathrm{THz}$ with relative bandwidth (RB) is up to $37.1 \%$, revealing that the LTCPC performs well attributing the success to the synergy of the metal and graphene when graphene present high $E_{\mathrm{f}}$. Meanwhile, the simulated ellipse angle (see Fig.4(b)) is close to $-45^{\circ}$ over working band, further indicating that the transmitted wave is very close to the circularly polarized wave with high-efficiency conversion property.

Since the graphene metasurfaces utilize the tuning elements to enhance the electric field and create gradual changes in phase and amplitude, it is expected that when graphene is ingeniously added into metamaterial, the tunable properties of graphene will result in varied transmitting responses. In detail, the extraordinary electronic and optical properties of graphene can be tuned by adjusting the doping level, leading to controlling the interaction between light and matter through regulating carrier density concentration inside the working resonant unit. In our work, due to the tunability of graphene, it is worth mentioning that the working frequency of LTCPC can be dynamically switched between the broadband and narrowband generated under the adjustment of the Fermi level of graphene.

The specific change is reflected in AR that the working band from single frequency to broadband, which occurs due to the decrease of the equivalent surface impedance of graphene 
generated by the enhancement of $E_{\mathrm{f}}$. This bandwidth change phenomenon combines the increment in amplitudes and an observable phase difference change between the two orthogonally transmitted waves. The electrostatic tunability of the proposed LTCPC was analyzed numerically via the AR curves of diverse $E_{\mathrm{f}}$ as shown in Fig.5. As the Fermi level is incremented up from $0.2 \mathrm{eV}$ to $1.1 \mathrm{eV}$, an expansion in the bandwidth is observed in the AR curves. The detailed circumstances about the frequency range of $3 \mathrm{~dB}$ AR bands located at $0.9355-1.033 \mathrm{THz}, 0.9498-1.2644 \mathrm{THz}$, $0.9498-1.3554 \mathrm{THz}$, and $0.9498-1.3827 \mathrm{THz}$, respectively by electrically doping the graphene $E_{\mathrm{f}}$ from $0.2 \mathrm{eV}$ to $1.1 \mathrm{eV}$ (the $\mathrm{RBs}$ are $9.9 \%, 28.4 \%, 35.2 \%$, and $37.1 \%$, respectively). One can obviously perceive that the graphene metasurfaces exhibiting a significant tuning capacity in the process of $E_{\mathrm{f}}$ change which can increase the efficiency of linear-to-circular polarization conversion, and also achieve dynamic tuning of the bandwidth of the proposed LTCPC. This phenomenon verifies that the method via controlling $E_{\mathrm{f}}$ of graphene to enhance and control the interaction between light and matter contributes to the design of tunable devices.
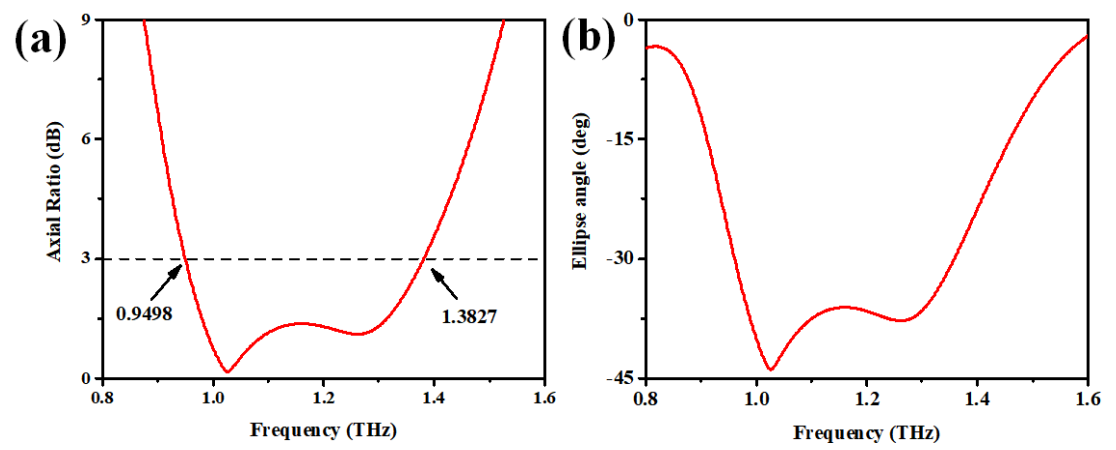

Fig.4. The AR and $\beta$ of the proposed metasurface when $E_{\mathrm{f}}=1.1 \mathrm{eV}$ : (a) the AR curve, and (b) the ellipse angle $\beta$ curve.

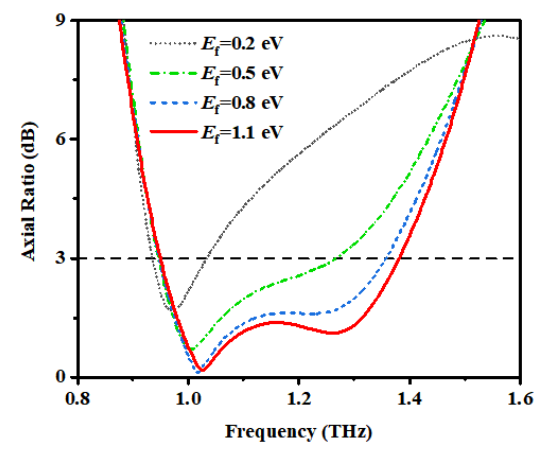

Fig.5. The AR curves of the proposed LTCPC with $E_{\mathrm{f}}$ runs from $0.2 \mathrm{eV}$ to $1.1 \mathrm{eV}$.

Given the above analyses from Fig.5 that the effect of graphene on the presentation of dynamic control is uniform in lower frequencies (such as $1.01 \mathrm{THz}$ ), and especially in the higher frequencies (such as $1.31 \mathrm{THz}$ ), there occurs the band extended region where resonance intensity gets improved effectively. Thus the physical mechanism of tunable functionality of this LTCPC is further explained based on the surface current distribution of the proposed structure plotted in Fig.6 when the $y$-polarized wave irradiates onto the proposed LTCPC, where the black arrows indicate the main current directions on the top, middle and bottom layers.

Fig.6(a) reveals the current distribution at resonant frequency $f=1.01 \mathrm{THz}$ in the case of $E_{\mathrm{f}}=0.2$ $\mathrm{eV}$, wherein the polarization conversion formed with $\mathrm{AR}$ is close to $0 \mathrm{~dB}$. First of all, an electric 
current is generated by the arrow-shaped gold resonance on the top layer, which then excites the currents of the resonance on the bottom layer. Therefore, the phase difference of $90^{\circ}$ is produced by the upper and bottom resonances. The resulting surface current fields serve as an excellent platform to confirm the linear-to-circular transformation of the proposed LTCPC. Compared to the situation of $1.01 \mathrm{THz}$ mentioned before, Fig.6(b) reveals the current distributions at $1.31 \mathrm{THz}$ in the case of $E_{\mathrm{f}}=0.2 \mathrm{eV}$. It can be interpreted that the current distribution is not strong enough to demonstrate the ability of linear-to-circular polarization at $1.31 \mathrm{THz}$, wherein there is only a weak current obviously and this is why there exists a narrow frequency band in $0.2 \mathrm{eV}$.

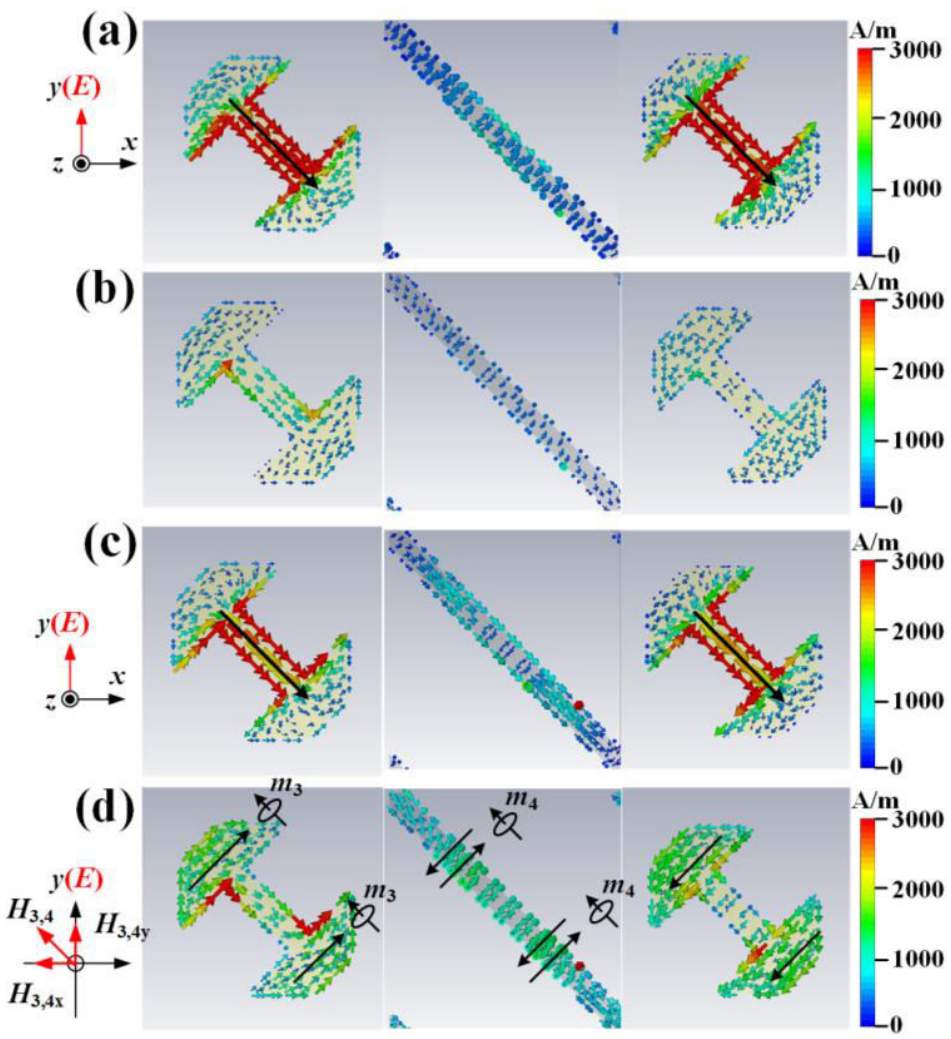

Fig.6.The surface current contributions on the top, middle and bottom mtasurfaces layers of the presented LTCPC for $y$-polarized EM waves at $1.01 \mathrm{THz}$ and $1.31 \mathrm{THz}$ when $E_{\mathrm{f}}=0.2 \mathrm{eV}$ and $E_{\mathrm{f}}=1.1 \mathrm{eV}$, respectively: (a) current contribution at $1.01 \mathrm{THz}$ when $E_{\mathrm{f}}=0.2 \mathrm{eV}$, (b) current contribution at $1.31 \mathrm{THz}$ when $E_{\mathrm{f}}=0.2 \mathrm{eV}$, (c) current contribution at $1.01 \mathrm{THz}$ when $E_{\mathrm{f}}=1.1 \mathrm{eV}$, and (d) current contribution at $1.31 \mathrm{THz}$ when $E_{\mathrm{f}}=1.1 \mathrm{eV}$.

As the gate voltage is adjusted to increase the Fermi level of graphene stripes, the working band is widened to different degrees. Particularly in the case of $E_{\mathrm{f}}=1.1 \mathrm{eV}$, the frequency band expands from one resonance frequency point to relatively wider bandwidth until another lowest AR frequency point was formed at $1.31 \mathrm{THz}$ with $\mathrm{AR}$ less than $3 \mathrm{~dB}$ ranging from $1.01 \mathrm{THz}$ to 1.31 THz. Since the general trend of current distributions depicted in Fig.6(c) is of the same situation of $E_{\mathrm{f}}=0.2 \mathrm{eV}$ at $1.01 \mathrm{THz}$, there is no need to repeat analyzing the surface currents. That is to say, the physical mechanism of the proposed LTCPC at $1.01 \mathrm{THz}$ is based on the same principle in both cases of $E_{\mathrm{f}}=0.2 \mathrm{eV}$ and $E_{\mathrm{f}}=1.1 \mathrm{eV}$. However, it is interesting that the surface current changes the main distribution area at $1.31 \mathrm{THz}$ in the case of $1.1 \mathrm{eV}$ as shown in Fig.6(d), due to the extraordinary ability in terms of tunability of graphene, its conductivity increases, thus the graphene also participates in the main current as resonances. In detail, the surface current distribution is transferred to both ends of the arrow-shaped like gold resonance, and it is of great significance that the surface current on the top layer is opposite to that on the middle layer, 
furthermore, the current on the middle layer is opposite to that on the bottom layer, which cause two circulating currents. Therefore, as per the coupling scheme, the antisymmetric currents induce two magnetic fields $\boldsymbol{H}_{3}$ and $\boldsymbol{H}_{\mathbf{4}}\left(\boldsymbol{m}_{\mathbf{3}}\right.$ and $\boldsymbol{m}_{\mathbf{4}}$ represent the magnetic dipoles respectively) in the direction perpendicular to the formed circulating currents. In particular, the ability of magnetic polarization gets improved reflected in the lower AR with the development of the intensity of currents which is attributed to the superposition of the double circulating currents. Hence, the induced magnetic field $\boldsymbol{H}_{\mathbf{3 , 4}}$ are paralleled to the electric field $\boldsymbol{E}$, which tally with a suitable coupling mechanism leading to the polarization conversion. On the contrary, there is no polarization conversion generating since the induced magnetic field $\boldsymbol{H}_{\mathbf{3 , 4} \mathbf{x}}$ are perpendicular to the electric field $\boldsymbol{E}$. Also, compared with the single surface current distribution at $1.01 \mathrm{THz}$ whether in $0.2 \mathrm{eV}$ or $1.1 \mathrm{eV}$ contributing to the resonance of single frequency point, the generation of superimposed multiple magnetic resonances at $1.31 \mathrm{THz}$ in the situation of $1.1 \mathrm{eV}$ make it an interesting way to expand bandwidth dependent on the overlapping frequency bands.

All in all, the graphene effect determined by the geometry mainly centers at the high-frequency band, and at these frequencies, the different degree of electromagnetic coupling between graphene and the original resonance unit at different $E_{\mathrm{f}}$ leads to the difference of current strength. The proximity of multiple magnetic resonances ascribed to the tunable optical property of graphene strongly enhances the transmission coefficient at high frequencies and assists in broadening the bandwidth of the LTCPC.
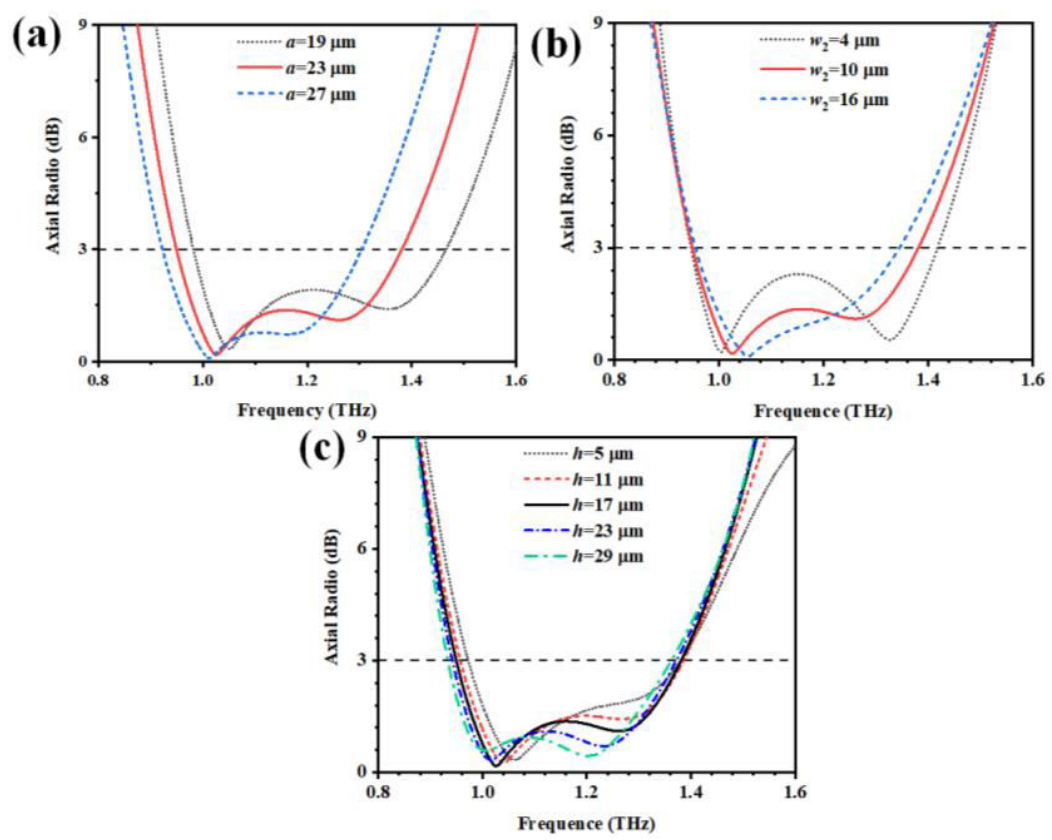

Fig.7. The AR curves of the proposed LTCPC for parameters $a, w_{2}$ and $h$ with different values when $E_{\mathrm{f}}=1.1 \mathrm{eV}$.

The structural parameters such as $a, b$, and $c$ which make up for the surface resonant unit are crucial to assess the sensitivity of the cell' s performance because the resonance tuning due to geometric difference plays a significant part in the plasmonic polarizer design. According to these measured results, we have obtained the AR curves of different $a$ in Fig.7.(a). It is apparent that the $3 \mathrm{~dB}$ AR band is slightly shifted to the high-frequency region with a little bandwidth broadening. Although the increase of $a$ from $19 \mu \mathrm{m}$ to $23 \mu \mathrm{m}$, and $27 \mu \mathrm{m}$ will broaden the relative bandwidth, it also leads to the elevation of AR making the curve unbalanced in the high-frequency part and prone to deteriorate further. After comparing the AR curves with different a in the criterion of low 
$\mathrm{AR}$ and wide bandwidth, $a=23 \mu \mathrm{m}$ is selected as the optimal value. Synchronously, the constituents of graphene geometries are the key parameters in the tunability principle of this LTCPC, where the width $w_{2}$ plays a major role in determining the effective area of graphene, so we have calculated the AR of varied $w_{2}$ in Fig.7.(b). In the process of adjusting the value of $w_{2}$, the equivalent impedance model will also change and there appears an expansion in the high-frequency band gradually due to the decrement of $w_{2}$ from $16 \mu \mathrm{m}$ to $4 \mu \mathrm{m}$ but there is also a trend that the AR will be unstable and exceed $3 \mathrm{~dB}$ in the middle-frequency part when $w_{2}=4 \mu \mathrm{m}$. On the premise of keeping a relatively wide bandwidth, the AR should be kept at a low and balanced level as far as possible. It can be seen that an optimal LTCPC performance can be realized in the case of $w_{2}=10 \mu \mathrm{m}$, which fits the comparatively suitable interaction required between graphene and impinging radiation. Next, we observed the effect of the thickness of the polyimide dielectric spacer on the AR performance. Fig.7(c) shows that when $h$ is varied from 5 $\mu \mathrm{m}$ to $29 \mu \mathrm{m}$, the AR curves of the transmitted wave vary subtly, while keeping the working bandwidth almost constant. Under the condition of maximum bandwidth, other factors should be taken into consideration, $h=17 \mu \mathrm{m}$ is selected as the optimal value after comparing the phase difference curves affected by thickness with each other.

In order to discuss the influence of losses in the polyimide, the diagram of amplitude for both co-polarization $\left(T_{\mathrm{xy}}\right)$ and cross-polarization $\left(T_{\mathrm{yy}}\right)$ at a different tangential $\operatorname{loss}(\tan \theta)$ of the polyimide which represents the loss more specifically is displayed in Fig.8.(a) and (b) It can be seen that when $\tan \theta$ is at a lower value, $T_{\mathrm{xy}}$ and $T_{\mathrm{yy}}$ are almost constant and remains close to each other whether in the case of 0.00027 or 0.0027 . However, further increase in $\tan \theta$ degrades the performance of the polarization converter as the amplitude decreased within the band when $\tan \theta$ grows to 0.027 , which amounts to a pronounced 100 times increased from the 0.00027 value initially stated. In spite of the slight decrease, the change is small and still remains within the allowable range. In all, with the enlargement of $\tan \theta$, the transmission coefficients are abated gradually but not significantly. In the light of the fact that the polyimide layers can give much higher losses than considered theoretically, the optimum $\tan \theta$ was selected as 0.00027 .
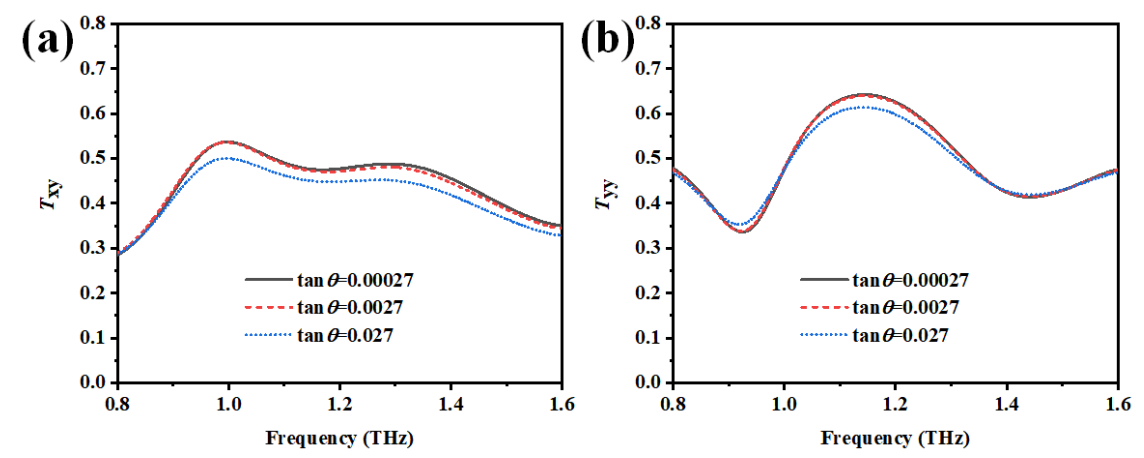

Fig.8. The simulated amplitude curves of the proposed LTCPC at different $\tan \theta$ : (a) the amplitude of co-polarization, and (b) the amplitude of cross-polarization.

\section{Conclusion}

In summary, a tunable LTCPC has been proposed in this paper based on three layers of anisotropic structures, where comprising two gold resonances on the top and bottom layers respectively, and the oblique graphene stripes on the middle layer. Applied a bias voltage, the adjusted electrical conductivity generated in the graphene material whose optical properties are 
tightly connected with the chemical potential has attracted considerable attention in tunable applications. As the gate voltage is simply adjusted to increase the $E_{\mathrm{f}}$ of graphene, the optimized band of AR lower than $3 \mathrm{~dB}$ located in 0.9498 to $1.3827 \mathrm{THz}$ (the RB is $37.1 \%$ ) when $E_{\mathrm{f}}$ is $1.1 \mathrm{eV}$ expands close to four times than that in $0.9355-1.033 \mathrm{THz}$ (the $\mathrm{RB}$ is $9.9 \%$ ) when $E_{\mathrm{f}}$ is $0.2 \mathrm{eV}$. Furthermore, the impacts of the structural parameters and the surface current contributions of the three metasurfaces are discussed concretely. Due to tunable properties, the graphene stripes are promised to possess the same function as the metal resonances to alter transmitting responses in higher $E_{\mathrm{f}}$, as a consequence, the effective bandwidth of the AR curve is increased to the required level. As a whole, the magnetically tunable ability endows the proposed LTCPC a broad application prospect in the manufacture of the $\mathrm{THz}$ imaging, sensing, and communication systems.

\section{Funding}

This work has no associated award funding.

\section{Conflicts of interest/Competing interests}

No conflict of interest exists in the submission of this manuscript, and the manuscript is approved by all authors for publication. I would like to declare on behalf of my co-authors that the work described was original research that has not been published previously, and not under consideration for publication elsewhere, in whole or in part. All the authors listed have approved the manuscript that is enclosed.

\section{Data Availability}

The datasets used or analyzed during the current study are available from the corresponding author on reasonable request.

\section{Author Contributions}

Xinlei Zhang, and Haifeng Zhang contributed to the novel idea of the study; Xinlei Zhang, Haining Ye and Yan Zhao contributed significantly to analysis and manuscript preparation; Haifeng Zhang performed the data analyses and wrote the manuscript.

\section{Ethics Approval}

I have read Plasmonics's Ethical Guidelines and state that I have not violated them in any way.

\section{Declarations}

Consent to Participate Informed consent was obtained from all individual participants included in the study.

Consent for Publication The participant has consented to the submission of the case report to the journal.

\section{References}

[1] V. K. Sharma, D. Madaan, and A. Kapoor, "Extremely short length and high extinction 
ratio plasmonic te mode pass polarizer," IEEE Photonics Technology Letters PP.7(2017) :1-1.

[2] D. Kim, "Performance uniformity analysis of a wire-grid polarizer in imaging polarimetry," Applied Optics 44.26(2005):5398-402.

[3] B. Lin, J. L. Wu, X. Y. Da, W. Li, and J. J. Ma, "A linear-to-circular polarization converter based on a second-order band-pass frequency selective surface," Applied Physics A 123.1(2017):43.

[4] Nasimuddin, X. Qing, and Z. N. Chen, "Compact Circularly Polarized Symmetric-Slit Microstrip Antennas," IEEE Antennas \& Propagation Magazine 53.4(2011):63-75.

[5] W. Zhao, L. Long, Y. Li, and C. Xi, "Metasurface Superstrate Antenna With Wideband Circular Polarization for Satellite Communication Application," IEEE Antennas \& Wireless Propagation Letters 15(2016):374-377.

[6] H. Qi, T. X. Wu, X. Zhu, R. Lu, and S. T. Wu, "Designs of wide-view and broadband circular polarizers," Optics Express 13.20(2005):8318.

[7] K. Sinchuk, R. Dudley, J. D. Graham, M. Clare, and M. A. Fiddy, "Tunable negative group index in metamaterial structures with large form birefringence," Optics Express 18.2(2010):463-472.

[8] M. Mutlu, A. E. Akosman, A. E. Serebryannikov, and E. Ozbay, "Asymmetric chiral metamaterial circular polarizer based on four U-shaped split ring resonators," Optics Letters 36.9(2011):1653-5.

[9] Y. Zhao, and A. Al ù, "Manipulating light polarization with ultrathin plasmonic metasurfaces," Physical Review B 84.20(2015):205428.

[10] S. Zhang, Y. S. Park, J. Li, X. Lu, W. Zhang, and X. Zhang, "Negative Refractive Index in Chiral Metamaterials," Physical Review Letters 102.2(2009):023901.

[11] J. Hao, Y. Yuan, L, Ran, T. Jiang, J. A. Kong, C. T. Chan, and L Zhou, "Manipulating Electromagnetic Wave Polarizations by Anisotropic Metamaterials," Physical Review Letters 99.6(2007):063908.

[12] D. R. Smith, J. B. Pendry and M. C. K. Wiltshire, "Metamaterials and Negative Refractive Index," Science 305.5685(2004):788-792 .

[13] N. K. Grady, J. E. Heyes, D. R. Chowdhury, Z. Yong, M. T. Reiten, A. K. Azad, A. J. Taylor, D. A. R. Dalvit, and H. T. Chen, "Terahertz metamaterials for linear polarization conversion and anomalous refraction," Science (New York, N.Y.) 340.6138(2013):1304-7 .

[14] Y. Li, J. Zhang, S. Qu, J. Wang, L. Zheng, Y. Pang, Z. Xu, and A. Zhang, "Achieving wide-band linear-to-circular polarization conversion using ultra-thin bi-layered metasurfaces," Journal of Applied Physics 117.4(2015):011129-4623.

[15] S. Y. Wang, W. Liu, and G. Wen, "Dual-band transmission polarization converter based on planar-dipole pair frequency selective surface," Scientific Reports 8.1(2018).

[16] Vinit, Singh, Yadav, Sambit, Kumar, and Ghosh, "Graphene-based metasurface for a tunable broadband terahertz cross-polarization converter over a wide angle of incidence," Applied Optics 57(29)(2018):8720-8726.

[17] X. Ma, W. Pan, H. Cheng, M. Pu, and X. Luo, "An Active Metamaterial for Polarization Manipulating," Advanced Optical Materials (2014)2.10.

[18] Wang. D, L. Zhang, Y. Gong, L. Jian, T. Venkatesan, and C. W. Qiu, "Multiband switchable terahertz quarter-wave plates via phase-change metasurfaces," IEEE Photonics Journal 
8(1)(2016):1-8.

[19] M. I. Katsnelson, K. S. Novoselov, and A. K. Geim, "Chiral tunnelling and the Klein paradox in graphene," Nature Physics 2.2(2006):620-625.

[20] H. Cheng, S. Chen, P. Yu, and J. Li, "Dynamically tunable broadband mid-infrared cross polarization converter based on graphene metamaterial," Applied Physics Letters 103.22(2013): 151107.

[21] J. Li, P. Yu, H. Cheng, W. Liu, Z. Li, B. Xie, S. Chen, and J. Tian, "Optical Polarization Encoding Using Graphene-Loaded Plasmonic Metasurfaces," Advanced Optical Materials (2016).

[22] H. Cheng, S. Q. Chen, P. Yu, J. x. Li, L. Deng, and J. G. Tian, "Mid-infrared tunable optical polarization converter composed of asymmetric graphene nanocrosses," Optics Letters (2013):38.9.

[23] A. K. Fahad, and C. Ruan, "A wideband terahertz transmissive polarization manipulator based on metasurfaces," Electronics 8(10)(2019):1068.

[24] H. F. Zhang, L. Zeng, G. B. Liu, and Huang, T, "Tunable linear-to-circular polarization converter using the graphene transmissive metasurface," IEEE Access PP (99)(2019):1-1.

[25] W. Lei, Y. Jiang, W. Jiao, W. Cao, and H. Zhang, "An ultra-broadband THz absorber based on graphene," International Symposium on Antennas IEEE(2017).

[26] Y. Gang, F. Ling, Y. Jin, C. Luo, and J. Yao, "Dynamically Electrically Tunable Broadband Absorber Based on Graphene Analog of Electromagnetically Induced Transparency," IEEE Photonics Journal 8.1(2017):1-8.

[27] X. He, X. Yang, S. Li, S. Shuang, and J. Jiang, "Electrically active manipulation of electromagnetic induced transparency in hybrid terahertz metamaterial," Optical Materials Express 6(10)(2016):3075-3085.

[28] Y. Gang, F. Ling, Y. Jin, C. Luo, and J. Yao, "Dynamically electrically tunable broadband absorber based on graphene analog of electromagnetically induced transparency," IEEE Photonics Journal 8(1)(2017):1-8.

[29] S. Luo, B. Li, A. Yu, J. Gao, X. Wang, and D. Zuo, "Broadband tunable terahertz polarization converter based on graphene metamaterial," Optics Communications 413(2018):184-189.

[30] S.A. Mikhailov and K. Ziegler, "New electromagnetic mode in graphene," Physical Review Letters 99(2007):016803.

[31] G. Xi, X. Y. Yu, W. P. Cao, Y. N. Jiang, and X. H. Yu, "Ultra-wideband circular-polarization converter with micro-split Jerusalem-cross metasurfaces," Chinese Physics B 25.12(2016):128102. 